\title{
Large airway obstruction by a chronic dissecting aortic aneurysm in the Marfan syndrome
}

\author{
MR Hargreaves, TJ Gilbert, R Pillai, G Hart
}

\begin{abstract}
Summary
We describe a patient with the Marfan syndrome who presented with an acute aortic dissection. She underwent composite graft replacement of the aortic root. She returned two years later with dyspnoea and stridor due to tracheal compression by a large chronic dissection of the thoracic aorta. Marfan patients are at risk of chronic dissection involving the remaining distal aorta and require regular noninvasive assessment following surgery.
\end{abstract}

Keywords: Marfan syndrome, aortic dissection

In the Marfan syndrome, defective fibrillin production predisposes to aortic dilatation and dissection. ${ }^{1}$ We report a Marfan patient who, two years after composite aortic root replacement for an acute dissection, presented with large airway obstruction due to progressive expansion of a chronic thoracic aortic dissection.

\section{Case report}

A 28-year-old woman presented as an emergency with severe chest pain. Her father had died suddenly of a ruptured aortic aneurysm aged 24 years. Her paternal grandmother also had died suddenly in her third decade.

Examination disclosed features of the Marfan syndrome. She was tall $\left(5^{\prime} 9^{\prime \prime}\right)$ and slender with arachnodactyly, joint laxity, a high arched palate and pectus carinatum. The lenses were not dislocated. In the left arm the blood pressure was $110 / 70 \mathrm{mmHg}$ and in the right $30 / 0 \mathrm{mmHg}$. Auscultation of the heart revealed a mid-systolic click and an early diastolic murmur of aortic regurgitation. Investigations included an electrocardiogram showing repolarisation changes; a chest X-ray with widening of the mediastinum; an echocardiogram which demonstrated dissection of the aortic root, moderate aortic regurgitation, prolapse of the posterior leaflet of the mitral valve and pericardial fluid without tamponade; computed tomography (CT) confirmed dissection of the aorta extending from the aortic root to the bifurcation.

At emergency surgery a proximal intimal aortic tear was found just distal to the aortic sinuses. Cardiopulmonary bypass was established with femoral arterial return and venous return from the right atrium. The pericardium was opened and a large bloody effusion was released. On opening the ascending aorta the aortic ring was abnormally dilated with obvious failure of coaptation of the valve cusps. The ascending aorta was replaced with a valved Haemoshield $^{\mathrm{TM}}$ conduit and the coronary arteries were re-implanted. Prior to suturing the Haemoshield ${ }^{\mathrm{TM}}$ conduit, both the proximal and distal ends of the aorta were reinforced using 'tissue' glue. This formaldehyde resin base approximates the adventitia and media rendering the sutured edges firmer. The aorta was clamped proximal to the innominate artery whilst the proximal anastomosis and the coronary re-implantation was carried out. During this period the patient was cooled.

The distal anastomosis was performed at the level of the innominate artery with the circulation arrested at $18^{\circ} \mathrm{C}$ and the aorta open. On re-establishing bypass there was no back flow from the false lumen. The histology of the aortic tissue confirmed cystic medial necrosis. Postoperative recovery was uneventful.

She remained well for two further years but then developed progressive breathlessness over three months and an inspiratory stridor. The chest X-ray showed marked dilatation of the thoracic aorta (figure 1). The intrathoracic trachea was compressed and pushed to the right. CT confirmed significant tracheal compression by a chronic dissecting aneurysm involving the transverse and descending thoracic aorta. The abdominal aorta was also involved in the aneurysmal process: a contrast-enhanced study suggested that the spinal arteries were maintained by the false lumen. Flow volume loops were consistent with large airway compression (figure 2). The risks associated with replacement of the thoracic and abdominal aorta were considered to be prohibitive. She died suddenly at home shortly afterwards. A post-mortem examination was not performed.

\section{Discussion}

In this patient the diagnosis of the Marfan syndrome was made from the positive family history and the involvement of two major systems, skeletal and cardiovascular. Although dissecting aneurysm and cardiovascular catastrophe are common in the Marfan syndronme, major airway obstruction due to a chronic dissecting aneurysm in this disease is rare. One reason for this may be that, until relatively recently, recovery from the initial acute dissection was uncommon. During the past 15 years improved peri-operative manage- 


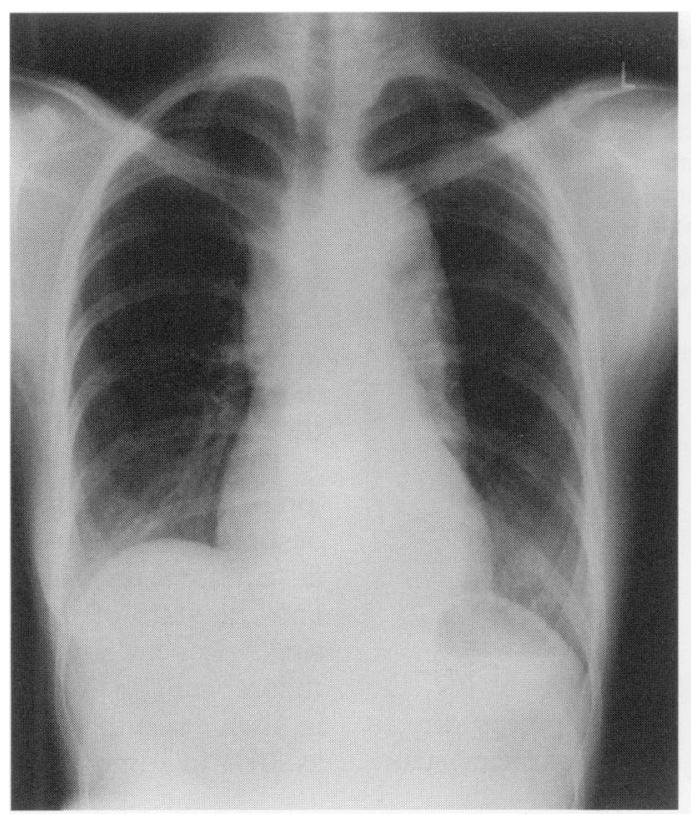

A

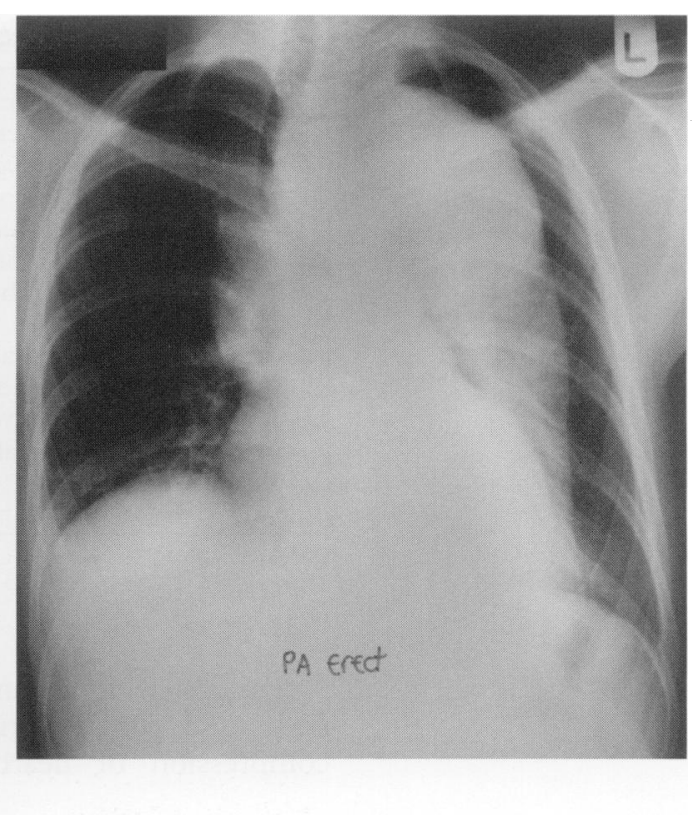

B

Figure 1 Chest radiograph (A) following aortic root replacement, and (B) two years later. A chronic dissecting aneurysm has resulted in significant tracheal deviation and compression

\begin{tabular}{l} 
Features of the Marfan syndrome \\
\hline Cardiovascular \\
- mitral and tricuspid valve prolapse \\
- mitral and tricuspid valve regurgitation \\
- aortic regurgitation \\
- infective endocarditis \\
- atrial septal aneurysm \\
- aortic root dilatation \\
- ventricular and supraventricular arrhythmias \\
Pulmonary \\
- apical blebs \\
- spontaneous pneumothorax \\
- kyphoscoliosis and subsequent restrictive \\
pulmonary defects \\
- pectus excavatum
\end{tabular}

Figure 2 Flow volume loops obtained two years following aortic root replacement. The early peak before the plateau on the expiratory limb of the flow-volume loop is characteristic of dynamic intrathoracic large airway obstruction. The upper tracing on the expiratory limb (……) shows predicted values

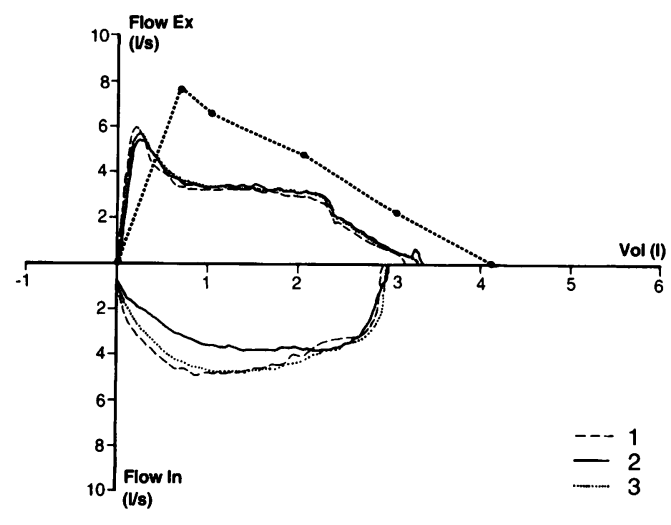

ment and surgical technique, particularly the utilisation of composite grafts with coronary artery implantation, have allowed increasing numbers of patients to survive beyond the short term. ${ }^{2}$ However, it has become apparent that, in some patients at least, aortic root

\section{Guidelines for follow-up of patients} with the Marfan syndrome

- patients with the Marfan syndrome should undergo interval transthoracic

echocardiography - the frequency of examination being determined by the absolute root diameter and rate of dilatation.

Prophylactic surgery is usually recommended when the aortic root diameter is more than $6 \mathrm{~cm}$

- in the Marfan syndrome pregnancy may be associated with rapid expansion of the aortic root. Echocardiographic surveillance should be increased during and immediately after pregnancy

- beta-blockade has been shown to reduce the rate of aortic root expansion

- following root replacement, the distal native aorta is at risk from repeated dissection, dilatation and rupture. The distal aorta may be assessed by either MRI or transoesophageal echocardiography. ${ }^{9}$ Patency and size, as well as flow in the false lumen, appears to have a bearing on prognosis. ${ }^{13,14} \mathrm{CT}$ scanning may be an alternative but involves repeated contrast and radiation exposure. Further surgery may be considered where there are symptoms or significant local dilatation

replacement may be a relatively short-term solution. The results from surgical follow-up studies suggests that there is often further aortic dilatation and aneurysm formation involving the false lumen distal to the graft, ${ }^{3,4}$ not only in patients with the Marfan syndrome but also in patients with dissection from other causes, eg, atheroma. In a recent publication, including follow-up magnetic resonance imaging (MRI) scanning of 28 patients following type A repair, 22 had demonstrable blood flow in a patent distal false lumen. ${ }^{5}$ 


\section{Summary/learning points}

- the Marfan syndrome may present with acute dissection of the ascending aorta. Without urgent surgical intervention death is inevitable from pericardial tamponade, aortic rupture or severe aortic regurgitation and heart failure

- replacement of the aortic root with a composite valve and graft prosthesis is the preferred treatment

- the remaining distal aorta remains at risk from chronic dissection and rupture. Regular followup and further elective aortic surgery may reduce the late mortality following aortic root replacement

Patients with an aneurysm involving the aortic arch may experience symptoms due to compression of nearby intrathoracic struc-

1 Hollister DW, Godfrey M, Sakai LW, Pyeritz RE. Immunohistologic abnormalities of the microfibrillar-fibre system in the Marfan syndrome. N Engl $\mathcal{F}$ Med 1990; 323: $152-9$.

2 Bentall H, De Bono A. A technique for complete replacement of the ascending aorta. Thorax 1968; 23: 338-9.

3 DeBakey ME, McCollum CH, Crawford ES, et al.

DeBakey ME, McCollum CH, Crawford ES, et al. Dissection and dissecting aneurysms of the aorta: twentysurgically. Surgery 1982; 92: 1118-34.

4 Crawford ES, Coselli JS, Svensson LG, Safi HG, Hess KR. Diffuse aneurysmal disease (chronic aortic dissection, Marfan, and mega aorta syndromes) and multiple aneurysm. Treatment by subtotal and total aortic replacement emphasising the elephant trunk operation. Ann Surg 1990; 211: $521-37$.

5 Moore NR, Parry AJ, Trottman-Dickinson B, Pillai R, Westaby $S$. Fate of the native aorta after repair of acute type A dissection: a magnetic resonance imaging study. Heart A dissection: a

6 Crawford ES, Saleh SA, Schuessler JS. Treatment of aneurysm of transverse aortic arch. $\mathcal{F}$ Thorac Cardiovasc Surg 1979; 78: 383-93.

7 Gaertner RA, Spencer FC, Bahnson HT. Current status of surgery of the lesions of the aortic arch. Prog Cardiovasc Dis 1962; 4: 373-90. tures. For example, patients may report hoarseness due to recurrent laryngeal nerve palsy, a superior vena cava syndrome or dysphagia due to oesophageal compression in addition to symptoms of large airway obstruction. $^{6-8}$ Why our patient did not experience any other compressive symptoms from her expanding aneurysm is unclear. Perhaps the relatively rapid expansion and her early death from presumed rupture simply did not allow time for the development of other symptoms.

This report emphasises the need for careful long-term follow-up including MRI and transoesophageal echocardiography to assess the false lumen, the presence of blood flow therein and the state of the graft and its anastomotic sites. ${ }^{9}$ Repeat surgery on the aorta can then, theoretically, be planned electively. ${ }^{10}$ However, in such cases the operative mortality is as high as $30 \%$ in some series. ${ }^{11,12}$

8 DeBakey ME, Noon GP. Aneurysms of the thoracic aorta Mod Concepts Cardiovasc Dis 1975; 44: $53-8$

9 Masani ND, Banning AP, Jones RA, Ruttley MST, Fraser AG. Follow-up of chronic thoracic aortic dissection: comparison of transoesophageal echocardiography and magnetic resonance imaging. Am Heart $f$ 1996; 131: magnetic

10 Taniguchi K, Nakano S, Matsuda $\mathrm{H}$, et al. Long-term survival and complications after composite graft replacement for ascending aortic aneurysm associated with aortic reflux. Circulation 1991; 84 (suppl 5): II131 -9.

11 Svenson LG, Crawford ES, Hess KR, Coselli JS, Safi HJ. Dissection of the aorta and dissecting aortic aneurysms. Circulation 1990; 82 (suppl IV): IV24-38.

12 Chirillo F, Marchiori MC, Andriolo L, et al. Outcome of 209 patients with aortic dissection: a 12 year multicentre experience. Eur Heart $₹$ 1990; 11: 311 -9.

13 Erbel R, Oelert H, Meyer J, et al. Effect of medical and surgical treatment on aortic dissection evaluated by TEE. Circulation 1993; 87: 1604-15.

14 Dinsmore RE, Willerson JT, Buckley MJ. Dissecting aneurysm of the aorta. Radiology 1972; 105: 567-72. 